\title{
The universal bed model for patient care improves outcome and lowers cost in cardiac surgery
}

\author{
Abbas Emaminia, MD, ${ }^{\mathrm{a}}$ Phillip C. Corcoran, MD, ${ }^{\mathrm{a}}$ Michael P. Siegenthaler, MD, ${ }^{\mathrm{a}}$ Melissa Means, MD, ${ }^{\mathrm{a}}$ \\ Sarah Rasmussen, MSN, RN, ${ }^{\mathrm{a}}$ Linda Krause, ACNP, ${ }^{\mathrm{a}}$ Damien J. LaPar, MD, MSc, ${ }^{\mathrm{b}}$ and \\ Keith A. Horvath, MD
}

\begin{abstract}
Objective: With the escalating demands to increase the efficiency and decrease the cost, innovations in postoperative cardiac surgical patient care are needed. The universal bed model is an innovative care delivery system that allows patient care to be managed in one setting from postoperation to discharge. We hypothesized that the universal bed model in the context of cardiac surgery would improve outcomes and efficacy.
\end{abstract}

\begin{abstract}
Methods: A total of 610 consecutive patients were admitted to the universal bed unit and prospectively entered into the Society of Thoracic Surgeons National Cardiac Database. Intensive care unit level of care was determined by acuity and staffing needs. Telemetry was employed from admission to discharge, and multidisciplinary rounds were conducted twice daily. Postoperative outcomes were recorded during hospital stay, and comparisons were made with the Society of Thoracic Surgeons National Cardiac Database using identical variables over the same period of time.
\end{abstract}

Results: Decreased ventilation time, intensive care unit and hospital stay, and reduction in the incidence of atrial fibrillation and infectious complications yielded a financial benefit in the universal bed group compared with the traditional model of admission. Stroke rate and in-hospital mortality were the same compared with regional and national centers. Compared with regional centers, there was an average cost savings between $\$ 6200$ and $\$ 9500$ per patient depending on the operation. Patient care satisfaction by independent survey was in the 99th percentile.

Conclusions: The universal bed patient care model allows for expedient and efficacious care as measured by decreased length of intensive care unit and hospital stay, improved postoperative outcomes, patient satisfaction, and cost savings. (J Thorac Cardiovasc Surg 2012;143:475-81)

The universal bed (UB) model, or acuity adaptable concept, is an innovative care delivery system that maintains patients in the same room from immediately postoperation to discharge, while adapting equipment, staff, and other resources according to a patient's level of acuity. In this system, there are no patient transfers and the required level of care is brought to the patient's bedside according to his or her needs. Hypothetically, this model leads to improved continuity of care and patient safety; diminished medical errors; increased patient, staff, and physician satisfaction; and cost savings. Although this model has been practiced in labor, delivery, recovery, and postpartum care for more than 3 decades, little is known about its efficacy and clinical

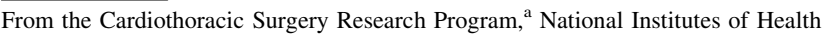
Heart Center at Suburban Hospital, Bethesda, Md; and Department of Surgery, ${ }^{\mathrm{b}}$ University of Virginia, Charlottesville, Va.

Disclosures: Authors have nothing to disclose with regard to commercial support.

Read at the 37th Annual Meeting of the Western Thoracic Surgical Association, June 22-25, 2011, Colorado Springs, Colorado.

Received for publication June 21, 2011; revisions received Aug 2, 2011; accepted for publication Oct 3, 2011; available ahead of print Dec 8, 2011.

Address for reprints: Keith A. Horvath, MD, Director, Cardiothoracic Surgery Research Program, NIH Heart Center at Suburban Hospital, 8600 Old Georgetown Road, Bethesda, MD (E-mail: horvathka@nhlbi.nih.gov). $0022-5223 / \$ 36.00$

Copyright (c) 2012 by The American Association for Thoracic Surgery doi:10.1016/j.jtcvs.2011.10.001
}

outcomes in a cardiac surgical setting. In this study, we hypothesized that the cardiac UB model will enhance patient satisfaction and diminish the rate of complications and cost of hospital admissions after cardiac surgery.

\section{MATERIALS AND METHODS \\ Patients}

This study was approved by the institutional review board of the $\mathrm{Na}$ tional Institutes of Health (NIH) Heart Center at Suburban Hospital. Prospectively, all patients who underwent cardiac operations between 2006 and 2009 were enrolled in the study, and data were collected using our institution's Society of Thoracic Surgeons (STS) National Adult Cardiac Surgery Database. Established STS database definitions were used for all preoperative variables, postoperative complications, and outcomes. Patients were stratified according to their primary procedure: coronary artery bypass graft (CABG), aortic valve repair or replacement, mitral valve repair or replacement, and any combinations of these procedures. All patient outcomes of interest were established a priori before data collection. Inhospital mortality was defined as patient deaths occurring before hospital discharge or within 30 days of operation. Postoperative variables were recorded, including arrhythmias, infection, stroke, myocardial infarction, total ventilation time, length of intensive care unit (ICU) and hospital stay, and readmissions to the hospital. The results were compared with similar variables from the STS national cardiac database as reported regionally and nationally over the same time period. Finally, we used the observed/ expected $(\mathrm{O} / \mathrm{E})$ ratio reported by the STS as a measure to compare our overall outcomes with the expected national outcome. In general, a smaller O/E ratio means better outcomes. 


$$
\begin{aligned}
& \text { Abbreviations and Acronyms } \\
& \begin{aligned}
\text { CABG } & =\text { coronary artery bypass graft } \\
\text { ICU } & =\text { intensive care unit } \\
\text { NIH } & =\text { National Institutes of Health } \\
\text { O/E } & =\text { observed/expected } \\
\text { STS } & =\text { Society of Thoracic Surgeons } \\
\text { UB } & =\text { universal bed }
\end{aligned}
\end{aligned}
$$

\section{Cardiac Universal Bed Room Features}

The cardiac UB unit at the NIH Heart Center became operational in 2006 and consists of 10 private rooms $\left(270-400 \mathrm{ft}^{2}\right.$ inclusive of the bathroom). All rooms accommodate all equipment necessary to provide all facets of critical care for patients after cardiac surgery. With the patient being transferred from the operating room, the room is set to "high acuity care," which is analogous to a traditional ICU room with equipment and monitoring required for the intensive care of a cardiac surgical patient. As the patients progress, unnecessary equipment and monitoring in the room are removed or discontinued to form a "progressive level care" similar to a traditional "step-down" room. Each room has its own bathroom with a shower and commode. Sufficient space is designed for families and nurses so that the presence of family members within the room does not interfere with medical staff function. There is a computer station inside each cardiac UB room for charting and recording. To enhance patient privacy and better sleep cycles, all rooms have mini-blinds and patients are allowed to darken the rooms at night when the acuity level is decreased.

\section{Admissions, Discharges, and Protocols in Universal Bed Model}

Patients have their preoperative visit with the surgeon and cardiovascular nurse practitioner a few days before the surgery, and the same nurses and physicians follow patients throughout their hospital stay. After surgery, patients are transferred directly to the UB unit and stay in the same room until they are discharged. The same staff nursing team gives care to the patients from admission to discharge.

Protocolized amiodarone is administered preoperatively and postoperatively unless contraindicated. Additional electrolyte replacement protocols and continuous insulin infusions are part of the standard postoperative orders. By working in conjunction with respiratory therapists, the goal is to extubate patients within 6 hours after surgery. Unless the patient is on inotropic support, pulmonary artery catheters are removed early on postoperative day 1 and patients are encouraged to ambulate. Once patients are extubated and intravenous inotropic or vasoactive medications are discontinued, patients are progressed to a lower acuity level. Typically on postoperative day 2 , chest tubes are removed and patients continue to ambulate, and by day 3 , plans are made for discharge. Multidisciplinary rounds are conducted twice per day for all patients regardless of their acuity level. The team comprises the cardiac surgeons, intensivist, clinical pharmacist, charge nurse, nurse practitioners, and registered nurses.

\section{Staff in Universal Bed Model}

Nursing staff assignment in the cardiac UB model is based on the acuity of patients. Nurse-to-patient ratio varies from 1:1 to 1:4, and nurses may be giving care to patients with different levels of acuity within their 12-hour shifts. Acuity level for patients is determined by their need of monitoring devices, mechanical ventilation, inotrope infusions, and overall hemodynamic stability. Appropriate staffing is practiced to keep the unit flexible in case a higher or lower level of care is required for any patient during his/her hospital stay.
We recruited a combination of cardiac ICU and telemetry nurses into the cardiac UB unit. The recruitment process included an interview in which details of the UB features were described. Nurses were made aware that the nurse-to-patient ratio varies according to the acuity of the patients. On employment in the cardiac UB, there are routine classes and orientation to train nurses to care for patients with all acuity levels.

\section{Families in Cardiac Universal Bed Model}

Families are encouraged to visit anytime except during nursing report at the change of shift. They are allowed in the patients' room soon after transfer from the operating room and can stay throughout the hospital course. Family members are welcome to participate and ask questions during rounds.

\section{Patient Satisfaction Survey}

A written patient satisfaction survey was randomly sent to patients via an independent survey company (Press Ganey Associates, Inc, South Bend, Ind) after discharge. Patients scored the unit according to their experience with admission processes, rooms, meals, nurses, tests and treatments, visitors and family, physicians, discharge, personal issues, and overall satisfaction.

\section{Statistical Analysis}

Primary outcomes of interest were in-hospital mortality, postoperative complications, ICU and length of hospital stay, and O/E ratio. Observed differences in patient characteristics and outcomes between study groups were compared. Categoric variables were compared using Pearson's chisquare or Fisher exact tests, and continuous variables were compared using the Student $t$ test for normally distributed data or the Mann-Whitney $U$ test for non-normally distributed data where appropriate. All categoric variables are expressed as within-group percentages, and continuous variables are expressed as means \pm standard error of the mean. All reported $P$ values are 2-tailed. Data analysis was performed using Predictive Analytics SoftWare version 18 (IBM Corp, Armonk, NY).

\section{RESULTS \\ Patient Characteristics}

Univariate analysis of patient risk factors is shown in Table 1. Over the 3-year study period, a total of 610 patients were prospectively enrolled in the study. Mean patient age was 69.7 years, and women accounted for $35 \%$ of all patients. Isolated CABG was the most commonly performed operation $(n=468,77 \%)$, followed by isolated valve operations $(\mathrm{n}=99,16 \%)$ and combined CABG/valve procedures $(n=43 \%)$ (Table 2$)$. Among valve operations, isolated aortic valve replacement/repair (61\%) was the most common procedure, followed by mitral valve repair $(35 \%)$ and mitral valve replacement $(4 \%)$. Patients undergoing cardiac operations within the study cohort presented with well-documented comorbid diseases: hypertension $(75 \%)$, peripheral arterial disease $(11.8 \%)$, dyslipidemia $(86 \%)$, renal failure on hemodialysis $(3.2 \%)$, diabetes $(24 \%)$, and low ejection fraction $(\mathrm{EF}<40 \%)(20 \%)$. Specifically, dyslipidemia, New York Heart Association class III and VI, preoperative arrhythmia, and rate of cardiogenic shock were significantly higher in patients admitted to the NIH Heart Center compared with regional and national centers. However, diabetes and reoperation rates were lower in 
TABLE 1. Demographic data of patients admitted to National Institutes of Health Heart Center and treated in the universal bed model

\begin{tabular}{|c|c|c|c|c|c|}
\hline & NIH Heart Center $(n=610)$ & Regional $(n=225,353)$ & $P$ value & National $(n=737,156)$ & $P$ value \\
\hline Age $($ mean \pm SEM $)$ & $69.7 \pm 2.82$ & $67.4 \pm 0.14$ & .3 & $66.2 \pm 0.08$ & .02 \\
\hline Age $>65 y$ & $426(70 \%)$ & $136,339(60.5 \%)$ & $<.0001$ & $446,400(60.6 \%)$ & $<.0001$ \\
\hline Family history of CAD* & $230(49.2 \%)$ & $27,612(31.6 \%)$ & $<.0001$ & $67,746(28.4 \%)$ & $<.0001$ \\
\hline NYHA class III & $190(31.2 \%)$ & $42,560(18.9 \%)$ & $<.0001$ & $140,165(19 \%)$ & $<.0001$ \\
\hline NYHA class VI & $98(16.1 \%)$ & $25,851(11.5 \%)$ & .0004 & $81,614(11.1 \%)$ & $<.0001$ \\
\hline Diabetes & $146(24 \%)$ & $69,247(30.7 \%)$ & .0004 & $221,146(30 \%)$ & .002 \\
\hline PAD & $82(13.4 \%)$ & $26,592(11.8 \%)$ & .2 & $89,933(12.2 \%)$ & .3 \\
\hline Dyslipidemia* & $403(86 \%)$ & $69,469(79.5 \%)$ & $<.0001$ & $190,119(79.7 \%)$ & $<.0001$ \\
\hline Hypertension & $455(75 \%)$ & $176,612(78.4 \%)$ & .03 & $570,979(77.5 \%)$ & .1 \\
\hline Renal failure & $20(3.2 \%)$ & $9014(4 \%)$ & .2 & $26,538(3.6 \%)$ & .5 \\
\hline $\mathrm{EF}<40$ & $121(20 \%)$ & $38,921(17.3 \%)$ & .08 & $129,318(17.5 \%)$ & .1 \\
\hline Arrhythmia & $81(13.4 \%)$ & $18,930(8.4 \%)$ & .05 & $64,132(8.7 \%)$ & $<.0001$ \\
\hline Cardiogenic shock & $35(5.8 \%)$ & $6535(2.9 \%)$ & $<.0001$ & $18,429(2.5 \%)$ & $<.0001$ \\
\hline MI $(\%)$ & $179(29.3 \%)$ & $63,518(28.2 \%)$ & .5 & $209,036(28.4 \%)$ & .5 \\
\hline Previous cardiac surgery & $40(6.5 \%)$ & $24,789(11 \%)$ & .0003 & $103,201(14 \%)$ & $<.0001$ \\
\hline
\end{tabular}

Comparison is made with the regional and national-scale centers. $P A D$, Peripheral arterial disease; $N Y H A$, New York Heart Association; $C A D$, coronary artery disease; $E F$, ejection fraction; $A F$, atrial fibrillation; $V T$, ventricular tachycardia; $V F$, ventricular fibrillation; $M I$, myocardial infarction. *Only for CABG and CABG/valve cases.

the NIH Heart Center cohort $(P<.0001$ and $P=.002$, respectively) (Table 1). Follow-up was 30 days or until discharge, and no patient was lost to follow-up.

\section{Postoperative Outcomes}

Differences in postoperative outcomes were observed between patients admitted to the UB unit and regional/ national patients (Table 3). Overall, for all major cardiac surgical procedures, in-hospital mortality was $3.6 \%$ in the UB cohort, which is similar to other regional/national centers. However, the incidence of major complications was significantly lower in the UB cohort $(P<.0003)$. Likewise, notably lower rates of postoperative arrhythmias, pneumonia, prolonged ventilation, and intra-aortic balloon pump placement were observed for the UB cohort $(P<.0001$ for all variables, $P=.0004$ for intra-aortic balloon pump placement). Most relevant to the hypothesis of this study, total postoperative ventilation hours, ICU stay, and total length of hospital stay were significantly lower in patients

TABLE 2. Procedure type and operative features for patients admitted to cardiac universal bed model

\begin{tabular}{|c|c|c|c|}
\hline & $\begin{array}{c}\text { CABG } \\
(n=468)\end{array}$ & $\begin{array}{c}\text { Valve } \\
(\mathbf{n}=99) \\
\end{array}$ & $\begin{array}{c}\text { CABG + valve } \\
(n=43)\end{array}$ \\
\hline Postoperative ventilation (h) & 6.6 & 6.8 & 14 \\
\hline Reintubation & $4.7 \%$ & $3.8 \%$ & $0 \%$ \\
\hline ICU stay (h) & 22.3 & 24.8 & 45.8 \\
\hline Readmission to ICU & $1.8 \%$ & $1.3 \%$ & $0 \%$ \\
\hline Postoperative atrial Fibrillation & $12.3 \%$ & $15.8 \%$ & $17 \%$ \\
\hline Sternal wound infection & $0 \%$ & $0 \%$ & $0 \%$ \\
\hline Mean hospital stay (d) & 6.6 & 6.9 & 10.7 \\
\hline $\operatorname{LOS}<6 \mathrm{~d}$ & $80.4 \%$ & $75.7 \%$ & $60.5 \%$ \\
\hline $\operatorname{LOS}>14 \mathrm{~d}$ & $1.8 \%$ & $4.6 \%$ & $7.4 \%$ \\
\hline Readmission to hospital & $5.7 \%$ & $11.1 \%$ & $10.9 \%$ \\
\hline
\end{tabular}

$L O S$, length of stay. admitted to the UB unit compared with regional/national centers $(P<.0001$ for all variables). The cost of hospitalization, reported by Maryland Healthcare Commission, was $\$ 6200$ to $\$ 9500$ lower than the regional average, depending on the type of operation, for the same period of time. The mean overall patient satisfaction score for the period of the study was 93.3 (99th percentile). The O/E ratio was significantly lower for patients admitted to the UB unit compared with regional and national outcomes $(0.68$ vs 1.08 vs $1.0, P<.0001)$.

\section{DISCUSSION}

The present study demonstrates the efficacy of the UB model in cardiac surgery. In a cohort of 610 patients, the cardiac UB model was associated with a significantly improved reduction in postoperative arrhythmias and pulmonary infections, and decreased length of ICU and hospital stay, all contributing to a markedly reduced cost of hospitalization. Furthermore, a high rate of patient satisfaction in postdischarge surveys is demonstrated in this model.

The UB/acuity-adaptable model of care is a "1-stop" care delivery concept that was introduced by the labor, delivery, recovery, and postpartum care process. In this model, patients stay in one room throughout their hospital stay, from admission to discharge, with the appropriate level of care brought to them. There are no transfers from one nursing care unit to another, and the UB room is changed accordingly to meet the level of acuity of individual patients. The concept was introduced in cardiothoracic surgery in the late 1970s and instituted in several community hospitals in the 1990s. ${ }^{1}$ At the beginning, one of the rationales for implementing this system was to compete with tertiary institutions, where cardiothoracic surgery was being practiced for many years. ${ }^{1}$ The primary focus was the 
TABLE 3. Postoperative outcome of patients admitted to National Institutes of Health Heart Center universal bed model of care

\begin{tabular}{|c|c|c|c|c|c|}
\hline & NIH Heart center $(n=610)$ & Regional $(n=225,353)$ & $P$ value & National $(n=737,156)$ & $P$ value \\
\hline Ventilation (h) (mean \pm SEM) & $26.3 \pm 1.07$ & $43.2 \pm 0.10$ & $<.0001$ & $41.9 \pm 0.05$ & $<.0001$ \\
\hline ICU h (mean \pm SEM) & $61.1 \pm 2.47$ & $110.5 \pm 0.23$ & $<.0001$ & $103.8 \pm 0.12$ & $<.0001$ \\
\hline Postoperative IABP (\%) & $81(0.4 \%)$ & $9110(4 \%)$ & $<.0001$ & $27,380(3.7 \%)$ & .0004 \\
\hline Complications ( $\%$ ) & $176(28.9 \%)$ & $120,049(53.3 \%)$ & $<.0002$ & $387,323(52.5 \%)$ & .0003 \\
\hline Sternal wound infection & $0(0 \%)$ & $837(0.4 \%)$ & .1 & $2948(0.4 \%)$ & .1 \\
\hline Stroke & $10(1.6 \%)$ & $4668(2.1 \%)$ & .4 & $15,690(2.1 \%)$ & .4 \\
\hline Perioperative MI & $6(1 \%)$ & $2640(1.2 \%)$ & .6 & $12,005(1.6 \%)$ & .2 \\
\hline Atrial fibrillation & $96(15.8 \%)$ & $69,924(31 \%)$ & $<.0001$ & $253,363(31.9 \%)$ & $<.0001$ \\
\hline Pneumonia & $7(1.2 \%)$ & $14,068(6.2 \%)$ & $<.0001$ & $37,490(5.1 \%)$ & $<.0001$ \\
\hline Readmission $(<30 \mathrm{~d})$ & $63(10.3 \%)$ & $31,887(14.2 \%)$ & .007 & $89,196(12.1 \%)$ & .1 \\
\hline Hospital stay $(\mathrm{d})($ mean $\pm \mathrm{SEM})$ & $8.5 \pm 0.34$ & $11.5 \pm 0.02$ & $<.0001$ & $11.6 \pm 0.01$ & $<.0001$ \\
\hline Hospital stay (d) (median) & 6.3 & 8.9 & $<.0001$ & 8.9 & $<.0001$ \\
\hline In-hospital mortality & $22(3.6 \%)$ & $10,946(4.9 \%)$ & .1 & $30,855(4.2 \%)$ & .4 \\
\hline $\mathrm{O} / \mathrm{E}$ ration & 0.68 & 1.08 & $<.0001$ & 1.0 & $<.0001$ \\
\hline
\end{tabular}

SEM, Standard error of the mean; $I A B P$, intra-aortic balloon pump; $A F$, atrial fibrillation; $M I$, myocardial infarction.

patient- and family-centered practice at the time, with little known about the outcome benefits. The first academic cardiac surgical program to use this model was fully functional in $2004 .^{2}$ To the best of the authors' knowledge, this is the first report on the cardiac surgical outcomes of the UB system.

Successful implementation of the UB model requires a combination of facility design and staffing support. Typically, different levels of acuity, from ICU to predischarge levels, should be accommodated; as a result, appropriate space needs to be provided for all potentially required cardiothoracic surgical ICU equipment, in addition to adequate space for caregivers, patients, and their families to function independently. Furthermore, rooms should meet the needs of "progressive care" patients who require telemetric monitoring and ease of ambulation. Our design of the cardiac UB adequately addresses these issues.

Although hospitals attempt to keep the cardiac surgical ICU and step-down floor on the same level and close to one another, sometimes they are located on different levels or patients may be distributed to several cardiac or noncardiac floors because of the unavailability of beds. One of the major advantages of the cardiac UB model is the elimination of patient transfers that usually occurs with changes in patient level of acuity. In this model, care is centered on patients and all services are brought to bedside, which results in minimized delays in the patient flow because of unavailability of beds at the correct level of care. To receive the appropriate care, patients may need to be transferred 3 to 6 times during an index hospitalization, and a typical nursing unit may transfer or discharge $40 \%$ to $70 \%$ of its patients every day. ${ }^{3}$ Transfers involve multiple hospital employees from nursing staff, pharmacy, dietary, and clerical staff, as well as physicians. ${ }^{3}$ With any transfer, there is a mandatory disruption in the continuity of care that results in a higher incidence of medical errors. ${ }^{4}$ Hendrich and colleagues $^{3}$ reported a $90 \%$ reduction in hospital transfers with the UB model that resulted in a 70\% decrease in medication errors. Fewer transfers also result in more patient satisfaction and lower stress level associated with a new location and team of caregivers. Leith ${ }^{5}$ demonstrated in a survey that up to $20 \%$ of patients leaving the ICU were highly distressed about the new level of care they received. The sudden decrease in the level of care encountered with the transfer from the ICU to telemetry is stressful for patients. ${ }^{5}$ Patients enjoy a greater sense of security being aware that they are given care by a single team of vigilant staff at all times. Our postdischarge satisfaction survey clearly highlights this issue.

We demonstrated that the incidence of pneumonia was significantly lower in this study compared with the national average and regional centers of the same size. In addition, no deep sternal wound infection was observed in our patients. Single-room occupancy in the UB model and protocolized care given to patients by the same nursing staff and respiratory therapists throughout the admission are potential contributing factors. Moreover, continuity of care in this model allows care providers to more quickly detect and treat infections should they occur. Nosocomial infection accounts for the main noncardiac complication after heart surgery, ${ }^{6}$ resulting in substantial morbidity, prolonged hospitalization, mortality, and economic burden. ${ }^{7}$ Decreasing the cost of care in the UB model is thought to be in part secondary to lower infection rates.

Although patients in this study had a higher rate of preoperative arrhythmias (including atrial fibrillation) compared with regional and national centers, postoperative arrhythmias were significantly lower. Prophylactic administration of amiodarone and continuation of this drug for 4 weeks is the mainstay of arrhythmia prevention in our practice. Although most atrial fibrillations occur on postoperative day 2, up to $43 \%$ of patients may experience more than 
1 episode on day 3 or within 2 days of the initial episode ${ }^{8}$ in what may be the "step-down" stay for most centers. A significantly lower incidence of postoperative atrial fibrillation in patients admitted to a UB unit is multifactorial, and prophylactic use of antiarrhythmic medications plays an important role. The continuous monitoring from the operating room to discharge may actually lead to overreporting of atrial fibrillation in a UB model. It also results in prompt detection and management of arrhythmias. Furthermore, abnormalities occurring during transfer of patients or shortly after they are placed in the new location may be missed, whereas the UB model eliminates this risk.

The single occupancy design of the UB room allows a higher level of privacy for patients and their families. In our practice, we encourage at least 1 member of the patient's family to stay with the patient as soon as he or she leaves the operating room until discharge. The patientand family-centered approach has reduced falls when patients start to ambulate and decreased the requirement of nurse hours per patient because families are involved in a portion of the care. ${ }^{9}$ Furthermore, this has resulted in high patient satisfaction in our experience.

Although there are many hypothetic benefits to the UB model, using this care delivery system is not without challenges. A single occupancy room with large square footage and all the equipment a cardiac surgical ICU needs is costly. In a North American analysis, authors have compared the cost to build a new wards with exclusively single-patient and double-patient occupancy and found that the former exceeds in cost with approximately $\$ 60,000 /$ patient $(\$ 182,400$ vs $\$ 122,550) .{ }^{10}$ This excludes the cost for equipment in a cardiac UB. This model of care delivery requires crosstraining of all nurses to be capable of functioning as ICU, progressive, and step-down nurses on a day-to-day basis. This potential additional cost may be offset by improved employee satisfaction and retention. However, with the UB model cost-saving trends, return of investment occurs within several years from implementation. From the nursing standpoint, the challenge of this model is the ability to adjust staffing ratios according to the patients' acuity levels. As patients are progressed, the ratios for the nursing personnel change accordingly. Also, it can be difficult to recruit critical care nurses to a unit where they may one day have a true ICU assignment or a very sick patient and another day have a telemetry 4-patient assignment. History dictates that ICU nurses are in critical care for the sickest patients, and once they are over the critical period, they are moved, so this represents another level of care they must assume. This is thoroughly discussed when potential staff are interviewed and trained. Caring for a patient from the critical care period to discharge has been voiced as a staff satisfier and may diminish the "burn out" experienced by nurses who only provide high acuity ICU level care. Our annual nursing surveys demonstrate a high satisfaction rate
$(>90 \%)$ among UB staff, and they have pointed out that the learning curve for switching from a traditional ICU to UB model is smooth and reasonable. Seeing patients throughout their stay and appreciating their progression from immediate postoperation to discharge are intriguing and rewarding to many of nurses who used to work in traditional ICU units before.

There are several incentives for hospitals to consider the UB approach. First is improved outcomes with the associated cost reduction for each individual hospital admission. Cardiovascular services, including cardiology and cardiac surgery, are considered the most profitable services for acute care hospitals. ${ }^{11}$ On the cardiac surgical side, it is shown that the strongest predictors of cost are hospital length of stay, number of hours spent in ICU, operating room time, and patient age. ${ }^{12}$ Second, despite the tendency toward decreased length of hospital stay, the appropriate level of care for cardiac surgical patients should be maintained. Patients in a cardiac UB model receive care from highly trained cardiac nurses throughout their admission. We have added to that the twice-daily multidisciplinary rounds of a team of surgeons and intensivists. Third, patients' satisfaction is a key in the current highly competitive era. An independent satisfaction survey showed that our cardiothoracic surgery unit was at the 99th percentile of overall patient satisfaction. Patients are particularly satisfied with the concept of remaining in one room for the whole duration of stay and the same group of nurses giving care to them. Finally, with a system that provides care across the continuum of care, patients who become unstable do not need to wait for an ICU bed to become available to deal with their crisis; rather, in the setting of their same rooms, their condition is upgraded to a higher acuity level. This issue is particularly important for cardiac surgical patients for whom time is of the essence in dealing with postoperative complications.

\section{Limitations}

Select limitations deserve discussion in this study. The sample size for this study was relatively small, and future studies are warranted with larger cohorts of patients. Furthermore, there was no previous model used at this hospital that could be used for a longitudinal comparison, and the data collected were from the beginning of this program.

\section{CONCLUSIONS}

The success of a cardiac UB model is evidenced by decreased morbidity rate and shorter duration of stay in the ICU and hospital, all resulting in a significant reduction in hospitalization cost. With comparable risk factor profiles, patients admitted to our cardiac UB model incurred lower postoperative ventilation time, ICU and hospital stays, and rate of atrial fibrillation and pneumonia. On the basis 
of this successful experience, we suggest expanding this model of care to other cardiac surgical programs.

\section{References}

1. Brown KK, Gallant D. Impacting patient outcomes through design: acuity adaptable care/universal room design. Crit Care Nurs Q. 2006;29:326-41.

2. Bush CA, Reisman D, Anstine L, Gallagher C, Davis R. The Ohio State University Richard M. Ross Heart Hospital: design and function of a specialty hospital in the academic environment. Am Heart Hosp J. 2005;3:120-7.

3. Hendrich AL, Fay J, Sorrells AK. Effects of acuity-adaptable rooms on flow of patients and delivery of care. Am J Crit Care. 2004;13:35-45.

4. Detsky ME, Etchells E. Single-patient rooms for safe patient-centered hospitals. JAMA. 2008;300:954-6.

5. Leith BA. Patients' and family members' perceptions of transfer from intensive care. Heart Lung. 1999;28:210-8.

6. Welsby IJ, Bennett-Guerrero E, Atwell D, White WD, Newman MF, Smith PK, et al. The association of complication type with mortality and prolonged stay after cardiac surgery with cardiopulmonary bypass. Anesth Analg. 2002;94:1072-8.

7. Michalopoulos A, Geroulanos S, Rosmarakis ES, Falagas ME. Frequency, characteristics, and predictors of microbiologically documented nosocomial infections after cardiac surgery. Eur J Cardiothorac Surg. 2006;29:456-60.

8. Mathew JP, Fontes ML, Tudor IC, Ramsay J, Duke P, Mazer CD, et al. A multicenter risk index for atrial fibrillation after cardiac surgery. JAMA. 2004;291:1720-9.

9. Chaudhury H, Mahmood A, Valente M. Nurses' perception of single-occupancy versus multioccupancy rooms in acute care environments: an exploratory comparative assessment. Appl Nurs Res. 2006;19:118-25.

10. The use of single patient rooms vs multiple occupancy rooms in acute care environments. Comparative first cost assessment of single and multiple occupancy patient rooms. Developed by Davis Langdon Adamson Construction Cost Planning and Management, Submitted to Coalition for Health Environments Research November 20, 2003. Available at: http://www.aia.org/SiteObjects/files/ 06_Comparative_Fir_Assessment.pdf. Accessed May 10, 2011.

11. Johnson J, Brown KK, Neal K. Designs that make a difference: the Cardiac Universal Bed model. J Cardiovasc Manag. 2003;14:16-20.

12. Hamilton A, Norris C, Wensel R, Koshal A. Cost reduction in cardiac surgery Can J Cardiol. 1994;10:721-7.

\section{Discussion}

Dr James Brevig (Everett, Wash). I work in Everett, Washington, at a community hospital. I thank the membership for the opportunity to discuss this presentation.

This is a difficult issue to study because it is hard to get a control group, which is evident to us from the presentation. Nevertheless, having worked in a similar model since 2004, which is when we opened our single-stay unit, a similar concept to the UB model, I am convinced this is a better model of care than the traditional model of care that involves a critical care unit and a variety of step-down, progressive care, or telemetry units. I am delighted to see this article and the concept getting some attention from our peers.

I would like to point out a couple of things that we as cardiac surgeons do not necessarily think about much, which is the process that goes on during the transfer of a patient and what the cost of that process is both to our patients and to our institution. I am going to remind us of a few of these steps.

Our patients typically recover from cardiac surgery in a critical care unit. At some point, the patient's nurse actually makes the decision that the patient is no longer critically ill, and at that point the patient stops getting critical care. The question then is what care is the patient getting? The patient is not getting telemetry care or critical care. Really, the patient is waiting for the next step, which is the surgeon to decide the patient is ready to progress. After that decision, they need to get a bed on another unit. Then the physical transfer, packing up the belongings, has to happen. The new nurse taking care of the patient will then get a report that involves an information transfer. Telemetry or step-down nurses typically work in 8-hour shifts, and so by this time it is probably a shift change, and so another nurse will get a report. If you think about the process, if I were going to design a process that was prone to error, this is the one I would design. This is the one we are using today.

One thing I would highlight from the presentation is the flexibility of this UB model that allows us to tailor the care to the patient's recovery, and so instead of erecting artificial barriers to the patient care, namely, which unit the patient happens to be in at the time, we can actually tailor the patient's care to the stage of his/her recovery. That works both ways by the way. The patient gets sick, needs a little inotropic or pressor support, volume, and respiratory support, and the patient is already in a critical care-capable room being taken care of by a critical care-trained nurse.

My last comment before I move on to my questions is that this should actually be presented as a quality of care improvement initiative and a process of care improvement initiative. It may well save money or it may not, but regardless, it is an improvement in the care we offer our patients.

You noted that you observed fewer complications than in your control groups, and I am not completely convinced of the validity of those controls, but I agree we need some kind of benchmark, and you used regional and national centers as your control groups. Which parts of those improvements do you think the universal care model was responsible for? You had a bunch of improvements in outcomes. You had a relatively short length of stay. Is there any causal relationship in any of this? You use the word "association" in your presentation.

Dr Emaminia. I could not agree more about the benefits that the UB model offers. In regard to the question, there are several outcomes and complications that we talked about, specifically, we focused on atrial fibrillation and pneumonia as 2 postoperative complications, and decreased length of ICU and hospital stay. For atrial fibrillation, as I said, we have treatment protocols and patients are prophylactically started on amiodarone before the operation. What the UB does to decrease the rate of postoperative arrhythmia is the continuity of care. When the patients are in the UB unit, they are being monitored throughout the stay, and any rhythm abnormality is proactively detected and prompted treated.

In terms of complications such as pneumonia, and in general postoperative infections, patients stay in 1 room and the same team of nurses and physicians are taking care of 1 patient. This approach decreases the contact that multiple house staff might have with a patient during hospital stay.

Dr Brevig. In your experience, are there any downsides to this? If there are members in the audience who like the presentation and concept, and want to institute this at their hospitals, what downsides did you find? What barriers did you have?

Dr Emaminia. There are multiple challenges in the way of starting a UB model. First may be the physical plant and that the new system should start from scratch, and the concept that there are separate ICUs and step-down units. Staffing issue is also important because within an 8- to 12-hour shift, there might be variable patient-tonurse ratios from $1: 1$ to $1: 4$, and nursing administration may have a hard time setting a fair and rational schedule for all their staff. 
Dr Brevig. How were you able to staff a 10-bed unit efficiently? One of the hurdles we had was that it was impossible to staff a small unit efficiently.

Dr Emaminia. Yes, challenges with staffing is one of the most important issues we have. Any UB model would have that issue, and we are currently working on that.

Dr Brevig. Thank you.

Dr Robert Cerfolio (Birmingham, Ala). Well presented. Congratulations to you and Dr Corcoran for bringing this to the forum. What about your design? You have shown that you have worldclass surgeons up there-you have Phil and a whole bunch of other great surgeons-and that you guys did better. Have you lost equipoise or could you now perform a randomized trial where you put half the patients into your standard of care and half the patients into a UB model? Would you be willing to do that now, Phil, or have you lost all equipoise to doing that?

Dr Corcoran. With regard to the UB model, our program, we are in a Certificate of Need state in Maryland, and the Maryland
State Health Care Commission controls absolutely every aspect of cardiovascular surgery. We are the ninth and for the foreseeable future probably the final program that has been opened in the state of Maryland. We are the only program in our local regional area that has maintained a UB model. One of the big issues is a nursing staffing issue, and that is something we are working through right now. There is no question about that. It would be difficult for us to go back. We do have a large series of ICUs because our hospital system does a lot of trauma surgery. It would be hard for us to put patients into a conventional ICU setting with step-down cardiac surgery at this point in time. It would require almost a reversal of our paradigm shift that we have had.

Dr Cerfolio. Then my only point is because they cannot, maybe someone in the audience can, and that is what the hospital administrators want to see to enact this. So if someone would do that, you would get up on the forum and change health care policy in the United States, and for members of the audience who have equipoise it would be easy to do. 